\title{
Some Studies in Sulfadiazine Incorporating Pyridine, Pyrimidine, Oxadiazole, and Azo Moieties Endowed with Pharmaceutical Potency
}

\author{
Sherihan A. El-Hadidya ${ }^{1, *}$ and Sraa Abu-Melhab ${ }^{2}$ \\ ${ }^{1}$ Department of Engineering Chemistry, Canal High Institute of Engineering and Technology, Sues, Egypt \\ ${ }^{2}$ Department of Chemistry, Faculty of Science, King Khalid University, Abha, Saudi Arabia \\ ${ }^{*}$ Corresponding author:E-mail: ice_princes52@yahoo.com \\ Tel: +201001678908
}

Received: 06-10-2019

\begin{abstract}
A set of substituted sulfadiazine compounds was prepared as cytotoxic and antitumor agents by using 4-amino- $N$-(pyrimidin-2-yl)benzenesulfonamide (1) as the starting material. Compound $\mathbf{1}$ was reacted with different reagents to give the corresponding sulfadiazines $\mathbf{2 - 1 8}$ and hydrozaones $\mathbf{1 9 a}-\mathbf{h}$ which were evaluated for their in vitro cytotoxicity versus four cancer cell lines. Compounds $3,5,19 \mathrm{~d}$ and $19 \mathrm{~h}$ were active against the tested cancer cells.
\end{abstract}

Keywords: Anticancer; Sulfonamide; Oxadiazole; Pyridine; Thiazole

\section{Introduction}

Sulfonamides have numerous biological actions, such as antibacterial, ${ }^{1}$ hypoglycemia, ${ }^{2}$ diuretic, ${ }^{3}$ anti-carbonic anhydrase ${ }^{3,4}$ and antithyroid. ${ }^{5}$ Recently, sulfonamides have been notified to exhibit fundamental antitumor action in vitro and/or in vivo. ${ }^{6,7}$ In addition, pyridine, pyrimidine, oxadiazole, and azo compounds are recognized to have various biological actions containing anticancer activity. ${ }^{8-15}$ So, the first goal in this realization is to synthesis several novel structures having anticancer action and the second goal is to exam the effect of the substitution of pyridine, pyrimidine, oxadiazole, and azo derivatives on the anticancer action and to research their structure action relationships.

\section{Experimental}

\section{1. Materials and Methods}

\section{1. 1. Chemicals and Reagents}

All the chemicals and solvents used in this study were obtained from Merck (Germany) and Sigma-Aldrich cheical company (Germany).

\section{1. 2. Instruments}

All melting points are measured on Gallenkamp electric melting point apparatus and are uncorrected. The Infrared spectra $v \mathrm{~cm}^{-1}(\mathrm{KBr})$ was measured on (Perkin Elmer Infrared Spectrophotometer Model 157, Grating). The ${ }^{13} \mathrm{C}$ and ${ }^{1} \mathrm{H}$ NMR spectra were run on Varian Spectrophotometer at $400 \mathrm{MHz}$ and $100 \mathrm{MHz}$ using TMS as an internal reference and DMSO- $d_{6}$ as solvent. The MS (EI) were measured on $70 \mathrm{eV}$ with Kratos MS equipment and/ or a Varian MAT 311 A Spectrometer at Cairo University, Giza, Egypt, and were carried on a GC-MS QP-100 EX Shimadzu (Japan). Elemental analyses (C, $\mathrm{H}$, and $\mathrm{N}$ ) were determined at the Microanalytical Center of Cairo University, Giza, Egypt. The results were in good agreement with the calculated values.

\section{2. Synthesis}

\section{2. 1. General Procedure for the Syntheses of (1,3-Dioxoisoindolin-2-yl)-N-(pyrimidin- 2-yl)benzenesulfonamide Derivatives 2-7}

A mix of $1(0.01 \mathrm{mmol})$ and anhydride derivatives namely; phthalic anhydride $(0.01 \mathrm{mmol}), 3$-nitrophthalic anhydride $(0.01 \mathrm{mmol}), 4$-nitrophthalic anhydride $(0.01 \mathrm{mmol}), 3,4,5,6$-tetrabromophthalic anhy- 
dride $(0.01 \mathrm{mmol}), 1,2,4$-benzenetricarboxylic anhydride (trimellitic anhydride), and 1,2,4,5-benzenetetracarboxylic dianhydride $(0.01 \mathrm{mmol})$ in $\mathrm{N}, \mathrm{N}$-dimethylformamide $(15 \mathrm{~mL})$ containing a few drops of triethylamine ( 3 drops) were boiled for $4 \mathrm{~h}$. The reaction mixture was left to cool to room temperature, and then put into ice-cold water. The insoluble precipitated product was purified and dried to yield sulfonamides $2-7$, respectively.

\section{4-(1,3-Dioxoisoindolin-2-yl)-N-(pyrimidin-2-yl)ben- zenesulfonamide (2)}

Yield (83\%), yellow powder, m.p. $288-290{ }^{\circ} \mathrm{C}$; IR $(\mathrm{KBr}): v / \mathrm{cm}^{-1} 3335(\mathrm{NH}), 1632(\mathrm{C}=\mathrm{O}$, imidic), 1575 $(\mathrm{C}=\mathrm{C}) ;{ }^{1} \mathrm{H}$ NMR (DMSO- $\left.d_{6}\right) \delta(\mathrm{ppm}): 6.76$ and $7.71(\mathrm{dd}$, $4 \mathrm{H}, \mathrm{Ar}-\mathrm{H} \mathrm{AB}$ system), 7.68-8.38 (m, 3H, Ar-H of pyrimidine ring), $9.13(\mathrm{~s}, 1 \mathrm{H}, \mathrm{NH})$; $\mathrm{MS} m / z(\%) 378\left(\mathrm{M}^{+}-2\right.$, 99.90), 347 (67.60), 189 (30.80), 151 (14.50); Anal. calcd. for $\mathrm{C}_{18} \mathrm{H}_{12} \mathrm{~N}_{4} \mathrm{O}_{4} \mathrm{~S}$ (380.06): C, 56.84; H, 3.18; N, 14.73 . Found: C, 56.79; H, 3.11; N, 14.72 .

\section{4-(4-Nitro-1,3-dioxoisoindolin-2-yl)-N-(pyrimidin-2-yl) benzenesulfonamide (3)}

Yield (89\%), white powder, m.p. $297-301{ }^{\circ} \mathrm{C}$; IR (KBr): $v / \mathrm{cm}^{-1} 3214(\mathrm{NH}), 1631(\mathrm{C}=\mathrm{O}$, imidic), 1551, 1337 $\left(\mathrm{NO}_{2}\right) ;{ }^{13} \mathrm{C}$ NMR (DMSO- $\left.d_{6}\right) \delta$ (ppm): $111.5,122.3(2 \mathrm{C})$, $123.6,127.8,128.2$ (2C), 134.1, 134.9, 135.7, 136.5, 138.3, $146.4,156.4(2 \mathrm{C}), 163.1(2 \mathrm{C}), 167.6$; $\mathrm{MS} m / z(\%) 423\left(\mathrm{M}^{+}-\right.$ 2, 4.18), 373 (63.15), 244 (100), 73 (20.34), 64 (47.39), 53 (14.39); Anal. calcd. for $\mathrm{C}_{18} \mathrm{H}_{11} \mathrm{~N}_{5} \mathrm{O}_{6} \mathrm{~S}$ (425.04): C, 50.82; H, 2.61; N, 16.46. Found: C, 50.93; H, 2.57; N, 16.41.

\section{4-(5-Nitro-1,3-dioxoisoindolin-2-yl)-N-(pyrimidin-2-yl) benzenesulfonamide (4)}

Yield (86\%), yellow powder, m.p. 226-228 C; IR (KBr): $v / \mathrm{cm}^{-1} 3361(\mathrm{NH}), 1721$ (C=O, imidic), 1540, 1380 $\left(\mathrm{NO}_{2}\right) ;{ }^{13} \mathrm{C}$ NMR (DMSO- $\left.d_{6}\right) \delta$ (ppm): $111.5,122.3(2 \mathrm{C}$ ), 123.3, 125.7, 128.2 (2C), 129.5, 133.6, 134.3, 136.5, 138.5, $152.1,156.4(2 \mathrm{C}), 163.1$ (2C), 167.6; MS $m / z(\%) 423\left(\mathrm{M}^{+}-\right.$ 2, 67.35), 373 (82.65), 244 (100), 73 (100); Anal. calcd. for $\mathrm{C}_{18} \mathrm{H}_{11} \mathrm{~N}_{5} \mathrm{O}_{6} \mathrm{~S}(425.04): \mathrm{C}, 50.82 ; \mathrm{H}, 2.61 ; \mathrm{N}, 16.46$. Found: C, $50.73 ; \mathrm{H}, 2.60 ; \mathrm{N}, 16.57$.

\section{$N$-(Pyrimidin-2-yl)-4-(4,5,6,7-tetrabromo-1,3-dioxoi-} soindolin-2-yl)benzenesulfonamide (5)

Yield (80\%), pale yellow powder, m.p. $257-260{ }^{\circ} \mathrm{C}$; IR (KBr): $v / \mathrm{cm}^{-1} 3360(\mathrm{NH}), 1632(\mathrm{C}=\mathrm{O}$, imidic), 1330 $\left(\mathrm{SO}_{2}\right), 760(\mathrm{C}-\mathrm{Br}) ;{ }^{13} \mathrm{C}$ NMR (DMSO- $\left.d_{6}\right) \delta(\mathrm{ppm}): 111.5$, 122.3 (2C), 126.1 (2C), 128.2 (2C), 129.4 (2C), 135.5, $136.5,138.9$ (2C), 156.4 (2C), 163.1 (2C), 167.6; MS m/z (\%) $691\left(\mathrm{M}^{+}, 12.3\right), 361$ (16.91), 360 (84.34), 330 (7.98), 314 (27.23), 221 (26.41), 185 (13.75), 164 (13.19), 138 (9.36), 103 (53.22), 95 (23.72), 79 (21.64), 75 (71.93), 64 (34.34), 53 (19.52); Anal. calcd. for $\mathrm{C}_{18} \mathrm{H}_{8} \mathrm{Br}_{4} \mathrm{~N}_{4} \mathrm{O}_{4} \mathrm{~S}$ (691.70): C, 31.06; H, 1.16; N, 8.05. Found: C, 31.02; H, $1.11 ; \mathrm{N}, 8.17$.
1,3-Dioxo-2-(4-(N-pyrimidin-2-ylsulfamoyl)phenyl) isoindoline-5-carboxylic acid (6)

Yield (89\%), white powder, m.p. $299-301{ }^{\circ} \mathrm{C}$; IR (KBr): $v / \mathrm{cm}^{-1}$ b. $3442(\mathrm{OH}$ of $\mathrm{COOH}), 3103(\mathrm{NH}), 1720$ (C=O, imidic); ${ }^{1} \mathrm{H}$ NMR (DMSO- $d_{6}$ ) $\delta$ (ppm): 7.51-8.35 (m, 7H, Ar-H), 9.01 (s, $1 \mathrm{H}, \mathrm{CH}$ of phthalimide), 8.31 and $8.46(2 \mathrm{~s}, 2 \mathrm{H}, 2 \mathrm{CH}), 9.13(\mathrm{~s}, 1 \mathrm{H}, \mathrm{NH}), 12.23(\mathrm{~s}, 1 \mathrm{H}$, $\mathrm{COOH}) ; \mathrm{MS} m / z(\%) 426\left(\mathrm{M}^{+}+2,15.26\right), 425\left(\mathrm{M}^{+}+1\right.$, 9.17), 361 (39.24), 360 (93.28), 330 (29.17), 314 (37.79), 221 (61.99), 185 (38.54), 164 (41.46), 138 (15.48), 103 (51.84), 95 (28.52), 79 (20.39), 75 (75.23), 64 (23.62), 53 (17.63); Anal. calcd. for $\mathrm{C}_{19} \mathrm{H}_{12} \mathrm{~N}_{4} \mathrm{O}_{6} \mathrm{~S}$ (424.05): C, 53.77; H, 2.85; N, 13.20. Found: C, 53.69; H, 2.91; N, 13.21 .

\section{Pyromellitimide Derivative 7}

Yield (84\%), white powder, m.p. $270-273{ }^{\circ} \mathrm{C}$; IR (KBr): $v / \mathrm{cm}^{-1} 3243(\mathrm{NH}), 1646(\mathrm{C}=\mathrm{O}$, imidic $), 1588$ $(\mathrm{C}=\mathrm{N}), 1579(\mathrm{C}=\mathrm{C}) ;{ }^{1} \mathrm{H}$ NMR (DMSO- $\left.d_{6}\right) \delta(\mathrm{ppm}): 7.59-$ $8.31(\mathrm{~m}, 14 \mathrm{H}, \mathrm{Ar}-\mathrm{H}), 10.18$ (s, 2H, 2CH), 9.15 (s, 2H, $2 \mathrm{NH}) ; \mathrm{MS} m / z(\%) 680\left(\mathrm{M}^{+}-2,22.35\right), 425$ (12.76), 361 (29.18), 360 (85.11), 330 (17.45), 314 (19.28), 221 (34.85), 185 (21.69), 164 (39.22), 138 (26.34), 103 (49.74), 95 (11.47), 79 (27.43), 75 (62.53), 64 (51.46), 53 (19.61); Anal. calcd. for $\mathrm{C}_{30} \mathrm{H}_{18} \mathrm{~N}_{8} \mathrm{O}_{8} \mathrm{~S}_{2}$ (682.07): C, 52.78; H, 2.66; N, 16.41. Found: C, 52.73; H, 2.72; N, 17.39 .

\section{4-(2,4-Dioxo-1,2-dihydroquinazolin-3(4H)-yl)-N-(py- rimidin-2-yl)benzenesulfonamide (8)}

A mix of $1(0.01 \mathrm{mmol})$ and isatoic anhydride $(0.01$ mmol) in $N, N$-dimethylformamide $(15 \mathrm{~mL})$ including a few drops of triethylamine ( 3 drops) were boiled for $4 \mathrm{~h}$. The reaction mixture was left to cool to room temperature, and then put into ice. The insoluble precipitated product was purified and dried to offer sulfonamide 8. Yield (79\%), white powder, m.p. $296-299{ }^{\circ} \mathrm{C}$; IR (KBr): $v / \mathrm{cm}^{-1} 3367$ (2NH), 1679 (CO, imidic); ${ }^{1} \mathrm{H}$ NMR (DMSO- $\left.d_{6}\right) \delta(\mathrm{ppm})$ : $4.28(\mathrm{~s}, 1 \mathrm{H}, \mathrm{NH}), 7.61-8.31(\mathrm{~m}, 11 \mathrm{H}, \mathrm{Ar}-\mathrm{H}), 9.13(\mathrm{~s}, 1 \mathrm{H}$, $\mathrm{NH}) ; \mathrm{MS} m / z(\%) 393\left(\mathrm{M}^{+}-2,41.46\right), 170$ (50.00), 84 (82.32), 56 (100); Anal. calcd. for $\mathrm{C}_{18} \mathrm{H}_{13} \mathrm{~N}_{5} \mathrm{O}_{4} \mathrm{~S}$ (395.07): C, 54.68; H, 3.31; N, 17.71. Found: C, 57.93; H, 3.27; N, 17.71 .

\section{2. 2. Syntheses of Diethyl 2-((4-(N-Pyrimidin- 2-ylsulfamoyl)phenylamino)methylene) malonate (9)}

To a solution of $1(0.01 \mathrm{mmol})$, diethyl 2-(ethoxymethylene)malonate $(0.01 \mathrm{mmol})$ was added in $\mathrm{N}, \mathrm{N}$-dimethylformamide $(25 \mathrm{~mL})$ including a few drops of trimethylamine. The mixture was boiled for $4 \mathrm{~h}$ and left to cool to room temperature, and then put into ice. The insoluble precipitated product was purified, dried to give compound 9. Yield (82\%), yellow powder, m.p. $181-183^{\circ} \mathrm{C}$; IR (KBr): $v / \mathrm{cm}^{-1} 3373(\mathrm{NH}), 1689$ and $1654(2 \mathrm{C}=\mathrm{O}$, ester); ${ }^{1} \mathrm{H}$ NMR (DMSO- $\left.d_{6}\right) \delta(\mathrm{ppm}): 1.24,1.28\left(2 \mathrm{t}, 6 \mathrm{H}, 2 \mathrm{CH}_{3}\right)$, $4.14,4.23\left(2 \mathrm{q}, 4 \mathrm{H}, 2 \mathrm{CH}_{2}\right), 8.35(\mathrm{~s}, 1 \mathrm{H}, \mathrm{CH}), 7.13-7.77(\mathrm{~m}$, 
7H, Ar-H), 9.07, 10.79 (2 s, $2 \mathrm{H}, 2 \mathrm{NH}) ;{ }^{13} \mathrm{C}$ NMR (DMSO- $\left.d_{6}\right) \delta$ (ppm): 26.5, 70.1, 110.5, 115.9 (2C), 117, 122.4, $129.7,130.8$ (2C), 132, 137.2, 142.6, 153.1, 155.4, 164.3, 177.2, 177.8; MS $m / z(\%) 422\left(\mathrm{M}^{+}+2,6.50\right), 232$ (29.10), 189 (99.90), 134 (22.90); Anal. calcd. for $\mathrm{C}_{18} \mathrm{H}_{20} \mathrm{~N}_{4} \mathrm{O}_{6} \mathrm{~S}$ (420.11): C, 51.42; H, 4.79; N, 13.33. Found: C, 53.71; H, $2.78 ; \mathrm{N}, 13.21$.

\section{2. 3. Syntheses of Ethyl 4-Oxo-6-(N-pyrimidin- 2-ylsulfamoyl)-1,4-dihydroquinoline-3- carboxylate (10)}

The cyclization reaction was performed by adding portions of $9(0.01 \mathrm{mmol})$ to boiling diphenyl ether (15 $\mathrm{mL}$ ). The reaction mix was boiled for $2 \mathrm{~h}$, cooled to room temperature, then added petroleum ether $(10 \mathrm{~mL})$. The obtained insoluble precipitated product was purified, washed with diethyl ether and dehydrated to offer sulfonamide 10. Yield (70\%), gray powder, m.p. charring at 277 ${ }^{\circ} \mathrm{C}$; IR (KBr): $v / \mathrm{cm}^{-1} 3219(\mathrm{NH}), 1681$ (C=O, ester), 1623 $(\alpha, \beta$-unsaturated $\mathrm{C}=\mathrm{O}), 1516(\mathrm{C}=\mathrm{C}) ;{ }^{1} \mathrm{H}$ NMR (DM$\left.\mathrm{SO}-d_{6}\right) \delta(\mathrm{ppm}): 1.30\left(\mathrm{t}, 3 \mathrm{H}, \mathrm{CH}_{3}\right), 4.31\left(\mathrm{q}, 2 \mathrm{H}, \mathrm{CH}_{2}\right), 7.24$ (s, $1 \mathrm{H}, \mathrm{C}_{2}-\mathrm{H}$ of quinoline), 7.69-7.85 (m, 6H, Ar-H), 8.94, 9.17 (2 s, $2 \mathrm{H}, 2 \mathrm{NH}) ; \mathrm{MS} m / z$ (\%) $375\left(\mathrm{M}^{+}+1,69.15\right), 310$ (100), 270 (86.17), 205 (94.68); Anal. calcd. for $\mathrm{C}_{16} \mathrm{H}_{14} \mathrm{~N}_{4} \mathrm{O}_{5} \mathrm{~S}$ (374.07): C, 51.33; H, 3.77; N, 14.97. Found: C, 51.39; H, 3.78; N, 14.81 .

\section{2. 4. yntheses of 3-(Hydrazinecarbonyl)-4-oxo- $\mathrm{N}$-(pyrimidin-2-yl)-1,4-dihydroquinoline- 6-sulfonamide (11)}

The cyclization reaction was performed by adding portions of $10(0.01 \mathrm{mmol})$ to boiling diphenyl ether (15 $\mathrm{mL}$ ). The mix was boiled for $1 \mathrm{~h}$, cooled to room temperature, then added petroleum ether $(10 \mathrm{~mL})$. The obtained insoluble precipitated product was purified, washed with diethyl ether and dehydrated. The resulting insoluble precipitate recrystallized from ethanol to yield sulfonamide 11. Yield (79\%), white powder, m.p. $122-125^{\circ} \mathrm{C}$; IR (KBr): $v / \mathrm{cm}^{-1} 3435\left(\mathrm{NH}_{2}\right), 3360(\mathrm{NH}), 1685$ (C=O, amidic), 1624 $(\alpha, \beta$-unsaturated $\mathrm{C}=\mathrm{O}), 1566(\mathrm{C}=\mathrm{C}) ;{ }^{1} \mathrm{H}$ NMR (DMSO- $\left.d_{6}\right) \delta(\mathrm{ppm}): 4.44\left(\mathrm{~s}, 2 \mathrm{H}, \mathrm{NH}_{2}\right), 7.36-8.38(\mathrm{~m}, 6 \mathrm{H}, \mathrm{Ar}-$ $\mathrm{H}), 8.38\left(\mathrm{~s}, 1 \mathrm{H}, \mathrm{C}_{2}-\mathrm{H}\right.$ of quinoline), 9.05, 9.18, $9.74(3 \mathrm{~s}, 3$ $\mathrm{H}, 3 \mathrm{NH}) ; \mathrm{MS} m / z$ (\%) $361\left(\mathrm{M}^{+}+1,1.20\right), 215$ (25.50), 103 (31.60), 43 (99.90); Anal. calcd. for $\mathrm{C}_{14} \mathrm{H}_{12} \mathrm{~N}_{6} \mathrm{O}_{4} \mathrm{~S}$ (360.06): C, 46.66; H, 3.36; N, 23.32. Found: C, 56.53; H, 3.27; N, 23.41 .

\section{2. 5. Syntheses of 2-(4-Oxo-6-(N-pyrimidin- 2-ylsulfamoyl)-1,4-dihydroquinoline-3- carbonyl)hydrazinecarbodithioic acid (12)}

To a solution of 11 (10 mmol) in ethanol containing a few drops of glacial acetic acid ( 4 drops) or in pyridine
(20 $\mathrm{mL})$, carbon disulphide $(10 \mathrm{~mL})$ was added and the reaction mixture was boiled for $4 \mathrm{~h}$. The solution was left to cool to room temperature and then put into ice. The insoluble precipitated was purified, dehydrated and recrystallized from ethanol to offer sulfonamide 12. Yield (81\%), white powder, m.p. $221-223{ }^{\circ} \mathrm{C}$; IR (KBr): $v / \mathrm{cm}^{-1} 3287$ and $3214(3 \mathrm{NH}), 1688(\mathrm{C}=\mathrm{O}$, amidic $), 1625(\alpha, \beta$-unsaturated $\mathrm{C}=\mathrm{O}), 1332(\mathrm{C}=\mathrm{S}) ;{ }^{1} \mathrm{H}$ NMR $\left(\mathrm{DMSO}-d_{6}\right) \delta(\mathrm{ppm})$ : 1.93 (s, 1H, SH), 7.61-8.24 (m, 6H, Ar-H), 9.02 (s, 1H, $\mathrm{C}_{2}$-H of quinoline), $9.22(\mathrm{~s}, 1 \mathrm{H}, \mathrm{NH}), 10.65,10.81(2 \mathrm{~s}, 2 \mathrm{H}$, 2NH); MS $m / z(\%) 432\left(\mathrm{M}^{+}-4,2.30\right), 378$ (21.20), 283 (99.90), 165 (31.80); Anal. calcd. for $\mathrm{C}_{15} \mathrm{H}_{12} \mathrm{~N}_{6} \mathrm{O}_{4} \mathrm{~S}_{3}$ (436.01): C, 41.27; H, 2.77; N, 19.25. Found: C, 41.23; H, $2.86 ; \mathrm{N}, 19.31$.

\section{2. 6. Syntheses of 3-(5-Mercapto-4,5-dihydro-} 1,3,4-oxadiazol-2-yl)-4-oxo- $\mathrm{N}$-(pyrimidin2-yl)-1,4-dihydroquinoline-6-sulfonamide (13)

A solution of compound 12 in ethanol $(25 \mathrm{~mL})$ including TEA ( 4 drops) was boiled for $4 \mathrm{~h}$. The formed precipitate was purified while heating, dehydrated and washed by ethanol to yield oxadiazole derivative 13 . Yield (85\%), white powder, m.p. $271-273{ }^{\circ} \mathrm{C}$; IR (KBr): $v / \mathrm{cm}^{-1} 3224(\mathrm{NH}), 2615(\mathrm{SH}), 1615(\alpha, \beta$-unsaturated $\mathrm{C}=\mathrm{O}), 1561(\mathrm{C}=\mathrm{N}), 1332(\mathrm{C}=\mathrm{S}) ;{ }^{13} \mathrm{C} \mathrm{NMR}\left(\mathrm{DMSO}-d_{6}\right) \delta$ (ppm): 111.5, 117.1, 118.2, 123.6, 131.1, 131.3, 133.7, $142.4,151.6,155.3,156$ (2C), 167.1, 167.6, 173.9; MS $m / z(\%) 402\left(\mathrm{M}^{+}, 45.10\right), 342$ (76.80), 122 (99.90), 43 (87.00); Anal. calcd. for $\mathrm{C}_{15} \mathrm{H}_{10} \mathrm{~N}_{6} \mathrm{O}_{4} \mathrm{~S}_{2}$ (402.02): C, 44.77; H, 2.50; N, 20.88. Found: C, 44.63; H, 2.66; N, 20.81 .

\section{2. 7. Syntheses of 3-(5-(Methylthio)-4,5- dihydro-1,3,4-oxadiazol-2-yl)-4-oxo-N- (pyrimidin-2-yl)-1,4-dihydroquinoline-6- sulfonamide (14)}

A mix of oxadiazole 13 (10 mmol), sodium hydroxide solution $(10 \mathrm{mmol})$, and methyl iodide $(10 \mathrm{mmol})$ was stirred in water $(15 \mathrm{~mL})$ for $14 \mathrm{~h}$. The forming thioether solution was removed by evaporation, and the residue collected by filteration, washed with water, dehydrated and recrystallized from ethanol to yield sulfonamide 14. Yield (85\%), gray powder, m.p. $172-175^{\circ} \mathrm{C}$; IR (KBr): $v / \mathrm{cm}^{-1}$ b. $3253(2 \mathrm{NH}), 1636(\alpha, \beta$-unsaturated $\mathrm{C}=\mathrm{O})$, and $1581(\mathrm{C}=\mathrm{N}) ;{ }^{1} \mathrm{H}$ NMR (DMSO- $\left.d_{6}\right) \delta(\mathrm{ppm})$ :

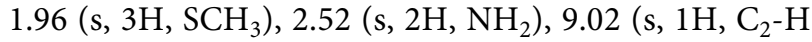
of quinoline), 7.60-8.24 (m, 3H, Ar-H), $10.66(\mathrm{~s}, 1 \mathrm{H}$, $\mathrm{NH}), 10.82$ (s, $1 \mathrm{H}, \mathrm{NH}) ; \mathrm{MS} m / z(\%) 413\left(\mathrm{M}^{+}+3,27.5\right)$, 258 (16.5), 248 (19.8), 233 (65.9), 209 (16.5), 207 (62.6), 143 (34.1), 115 (26.4), 77 (54.1), 76 (14.3), 69 (11.0), 65 (13.2), 51 (45.1), 50 (14.3); Anal. calcd. for $\mathrm{C}_{16} \mathrm{H}_{12} \mathrm{~N}_{6} \mathrm{O}_{4} \mathrm{~S}_{2}$ (416.04): C, 46.15; H, 2.90; N, 20.18. Found: C, 46.33; H, $2.86 ; \mathrm{N}, 20.01$. 


\section{2. 8. Syntheses of 4-Oxo-N-(pyrimidin-2-yl)- 3-(5-thioxo-4-((para-tolylamino)methyl)- 4,5-dihydro-1,3,4-oxadiazol-2-yl)-1,4- dihydroquinoline-6-sulfonamide (15)}

A mix of $13(10 \mathrm{mmol})$ and para-touluidine (10 $\mathrm{mmol}$ ) was boiled in ethanol $(30 \mathrm{~mL})$ with $30 \%$ formaldehyde $(20 \mathrm{mmol})$ for $3 \mathrm{~h}$. The resulting insoluble precipitate was recrystallized from ethanol to yield sulfonamide 15. Yield (72\%), brown powder, m.p. $193-195{ }^{\circ} \mathrm{C}$; IR (KBr): $v / \mathrm{cm}^{-1} 3325(\mathrm{NH}), 2969(\mathrm{CH}), 1635(\alpha, \beta$-unsaturated $\mathrm{C}=\mathrm{O}), 1610(\mathrm{C}=\mathrm{N}), 1267(\mathrm{C}=\mathrm{S}) ;{ }^{1} \mathrm{H}$ NMR (DMSO- $\left.d_{6}\right) \delta(\mathrm{ppm}): 2.16\left(\mathrm{~s}, 3 \mathrm{H}, \mathrm{CH}_{3}\right), 4.97\left(\mathrm{~s}, 1 \mathrm{H}, \mathrm{N}^{-\mathrm{CH}_{2}-}\right.$ $\mathrm{NH}), 5.85\left(\mathrm{~s}, 2 \mathrm{H}, \mathrm{N}-\mathrm{CH}_{2}-\mathrm{NH}\right), 7.60-8.24(\mathrm{~m}, 10 \mathrm{H}, \mathrm{Ar}-\mathrm{H})$, $8.39\left(\mathrm{~s}, 1 \mathrm{H}, \mathrm{C}_{2}-\mathrm{H}\right.$ of quinoline), $9.15(\mathrm{~s}, \mathrm{H}, \mathrm{NH}), 11.74$ (s, $\mathrm{H}, \mathrm{NH}) ; \mathrm{MS} m / z$ (\%) $521\left(\mathrm{M}^{+}, 17.87\right), 415$ (45.76), 401 (43.51), 301 (21.22), 220 (76.16), 158 (68.25), 120 (28.15), 100 (50.12), 77 (84.41), 76 (19.35), 69 (12.46), 65 (27.35), 50 (28.30); Anal. calcd. for $\mathrm{C}_{23} \mathrm{H}_{19} \mathrm{~N}_{7} \mathrm{O}_{4} \mathrm{~S}_{2}$ (521.09): C, 52.96; H, 3.67; N, 18.80. Found: C, 52.93; H, 3.65; N, 18.71 .

\section{2. 9. Syntheses of 2-Cyano-N-(4-(N-pyrimidin- 2-ylsulfamoyl)phenyl)acetamide (16)}

A mix of $1(0.01 \mathrm{mmol})$ and ethyl cyanoacetate $(0.01$ $\mathrm{mmol}$ ) was boiled in $N, N$-dimethylformamide $(25 \mathrm{~mL})$ for $4 \mathrm{~h}$. The reaction mixture was cooled to room temperature and then put into ice. The precipitated insoluble precipitated product was purified, dried and recrystallized from ethanol to yield sulfonamide 16. Yield (89\%), pale yellow powder, m.p. $237-239^{\circ} \mathrm{C}$; IR (KBr): $v / \mathrm{cm}^{-1} 3357(\mathrm{NH})$, $2155(\mathrm{CN}), 1647$ (C=O, amidic); ${ }^{1} \mathrm{H}$ NMR (DMSO- $\left.d_{6}\right) \delta$ (ppm): 4.25 (s, $\left.2 \mathrm{H}, \mathrm{CH}_{2}\right), 7.62-8.26(\mathrm{~m}, 3 \mathrm{H}, \mathrm{Ar}-\mathrm{H}$ and Ar-H of pyrimidine), $10.94,11.19(2 \mathrm{~s}, 2 \mathrm{H}, 2 \mathrm{NH}) ;{ }^{13} \mathrm{C}$ NMR (DMSO- $\left.d_{6}\right) \delta(\mathrm{ppm}): 26.5,112.7,117,122.4,125.8$ (2C), 129.7 (2C), 133.9, 141.2, 143.6, 153.1, 164.3; MS m/z (\%) $317\left(\mathrm{M}^{+}, 68.74\right), 218$ (86.32), 126 (88.42), 73 (100); Anal. calcd. for $\mathrm{C}_{13} \mathrm{H}_{11} \mathrm{~N}_{5} \mathrm{O}_{3} \mathrm{~S}$ (317.06): C, 49.21; $\mathrm{H}, 3.49$; $\mathrm{N}, 22.07$. Found: C, 49.19; H, 3.52; N, 22.81.

\section{2. 10. Syntheses of 4-Amino-2-oxo-N- (pyrimidin-2-yl)-1,2-dihydroquinoline-6- sulfonamide (17)}

Compound $1(0.01 \mathrm{mmol})$ was boiled in $\mathrm{N}, \mathrm{N}$-dimethylformamide $(30 \mathrm{~mL})$ including trimethylamine for 4 $\mathrm{h}$. The reaction mixture was cooled to room temperature; then put into ice. The insoluble precipitated product was purified, dried and recrystallized from ethanol to offer sulfonamide 17. Yield (90\%), yellow crystals, m.p. 210-213 ${ }^{\circ} \mathrm{C}$; IR (KBr): $v / \mathrm{cm}^{-1} 3357(\mathrm{NH}), 1713(\mathrm{C}=\mathrm{O}$, amidic), $1651(\alpha, \beta$-unsaturated $\mathrm{C}=\mathrm{O})$, and $1584(\mathrm{C}=\mathrm{C}) ;{ }^{1} \mathrm{H}$ NMR $\left(\mathrm{DMSO}-d_{6}\right) \delta(\mathrm{ppm}): 4.82\left(\mathrm{~s}, 2 \mathrm{H}, \mathrm{NH}_{2}\right), 8.33\left(\mathrm{~s}, 1 \mathrm{H}, \mathrm{C}_{2}-\mathrm{H}\right.$ of quinoline), 7.58-8.34 (m, $6 \mathrm{H}, \mathrm{Ar}-\mathrm{H}), 8.85(\mathrm{~s}, \mathrm{H}, \mathrm{NH})$, 10.15 (s, H, NH); MS $m / z$ (\%) $322\left(\mathrm{M}^{+}-5,52.46\right), 320$ (83.59), 231 (100), 135 (35.66); Anal. calcd. for
$\mathrm{C}_{13} \mathrm{H}_{11} \mathrm{~N}_{5} \mathrm{O}_{3} \mathrm{~S}$ (317.06): $\mathrm{C}, 49.21 ; \mathrm{H}, 3.49 ; \mathrm{N}, 22.07$. Found: C, $49.25 ; \mathrm{H}, 3.43 ; \mathrm{N}, 22.01$.

\section{2. 11. Syntheses of 3-Oxo-N-(4-(N-pyrimidin-2- ylsulfamoyl)phenyl)butanamide (18)}

A mix of $1(0.01 \mathrm{mmol})$ and ethyl acetoacetate $(10$ $\mathrm{mmol}$ ) was boiled in $N, N$-dimethylformamide $(25 \mathrm{~mL})$ including a few drops of trimethylamine for $3 \mathrm{~h}$. The reaction mixture was cooled to room temperature and then put into ice. The precipitated solid product was purified, dried and recrystallized from ethanol to offer sulfonamide $\mathbf{1 8 .}$

Yield (95\%), white powder, m.p. $228-230{ }^{\circ} \mathrm{C}$; IR (KBr): $v / \mathrm{cm}^{-1}$ b. $3315(2 \mathrm{NH}), 1725(\mathrm{C}=\mathrm{O}$, amidic $), 1689$ $(\mathrm{C}=\mathrm{O}), 1567(\mathrm{C}=\mathrm{N}), 1549(\mathrm{C}=\mathrm{C}) ;{ }^{1} \mathrm{H}$ NMR $\left(\right.$ DMSO- $\left.d_{6}\right) \delta$ (ppm): 2.31 (s, $\left.3 \mathrm{H}, \mathrm{CH}_{3}\right), 3.33$ (s, $\left.2 \mathrm{H}, \mathrm{CH}_{2}\right), 7.45-8.21(\mathrm{~m}$, $7 \mathrm{H}, \mathrm{Ar}-\mathrm{H}), 8.47(\mathrm{~s}, 1 \mathrm{H}, \mathrm{NH}), 10.16(\mathrm{~s}, 1 \mathrm{H}, \mathrm{NH}) ;{ }^{13} \mathrm{C} \mathrm{NMR}$ $\left(\mathrm{DMSO}-d_{6}\right) \delta(\mathrm{ppm}): 25,29.1,38.6,71.3,110.2,117,122.4$, 133.9, 137.2, 149.8, 152 (2C), 164.3, 177.8; MS $m / z(\%) 335$ $\left(\mathrm{M}^{+}+1,9.80\right), 180$ (87.00), 150 (51.10), 91 (99.90); Anal. calcd. for $\mathrm{C}_{14} \mathrm{H}_{14} \mathrm{~N}_{4} \mathrm{O}_{4} \mathrm{~S}$ (334.07): C, 50.29; $\mathrm{H}, 4.22 ; \mathrm{N}$, 16.76. Found: C, 50.25; H, 4.34; N, 16.78.

\section{2. 12. General Procedure for the Syntheses of Aryl Hydrazone Derivatives 19a-h}

A well stirred solution of aromatic amines $(20 \mathrm{mmol})$ in concentrated $\mathrm{HCl}(6 \mathrm{~mL})$ and water $(4 \mathrm{~mL})$ was cooled in an ice bath and diazotized with a solution of sodium nitrite $(1.39 \mathrm{~g}, 20 \mathrm{~mL})$ in water $(5 \mathrm{~mL})$.

The above cooled diazonium salt solution was added drop wise to a well stirred cooled solution of $\mathbf{1}$ in pyridine $(10 \mathrm{~mL})$. The reaction mixture was stirred for $1-2 \mathrm{~h}$ until giving a complete coupling reaction. The crude insoluble precipitate was purified, dehydrated well and recrystallized from ethanol to offer compounds $19 \mathbf{a}-\mathbf{h}$, respectively.

(E)-3-Oxo-N-(4-(N-pyrimidin-2-ylsulfamoyl)phenyl)-2(2-(thiazol-2-yl)hydrazono)butanamide (19a)

Yield (80\%), dark gray powder, m.p. charring at 271 ${ }^{\circ} \mathrm{C}$; IR (KBr): $v / \mathrm{cm}^{-1}$ b. $3250(3 \mathrm{NH}), 1700(\mathrm{C}=\mathrm{O}$, amidic), $1663(\mathrm{C}=\mathrm{O}), 1550(\mathrm{~N}=\mathrm{N}) ;{ }^{1} \mathrm{H}$ NMR (DMSO- $\left.d_{6}\right) \delta(\mathrm{ppm})$ : $2.23\left(\mathrm{~s}, 3 \mathrm{H}, \mathrm{CH}_{3}\right), 6.57(\mathrm{~d}, 1 \mathrm{H}, \mathrm{CH}$ of thiazole ring), 7.04 (d, $1 \mathrm{H}, \mathrm{CH}$ of thiazole ring), $7.82(\mathrm{~d}, 2 \mathrm{H}, \mathrm{Ar}-\mathrm{H}), 7.93(\mathrm{~d}$, $2 \mathrm{H}, \mathrm{Ar}-\mathrm{H}$ ), 7.65-8.39 (m, 3H, Ar- $\mathrm{H}$ of pyrimidine), 8.82 (s, H, NH), 9.04 (s, H, NH), $10.16(\mathrm{~s}, \mathrm{H}, \mathrm{NH}) ;{ }^{13} \mathrm{C}$ NMR $\left(\mathrm{DMSO}-d_{6}\right) \delta(\mathrm{ppm}): 26.5,114.7,117,122.4,124.1(2 \mathrm{C})$, 127.4 (2C), 131.5, 133.9, 141.6, 143.4, 145.6, 153.1, 164.3, 169, 177.8; MS $m / z(\%) 445\left(\mathrm{M}^{+}, 41.23\right), 366$ (12.34), 287 (24.18), 158 (12.56), 98 (59.76), 84 (69.34), 79 (78.46), 76 (83.76); Anal. calcd. for $\mathrm{C}_{17} \mathrm{H}_{15} \mathrm{~N}_{7} \mathrm{O}_{4} \mathrm{~S}_{2}$ (445.06): C, 45.83; H, 3.39; N, 22.01. Found: C, 45.91; H, 3.33; N, 22.01 .

(E)-2-(2-(1,5-Dimethyl-3-oxo-2-phenyl-2,3-dihydro-1Hpyrazol-4-yl)hydrazono)-3-oxo- $\mathrm{N}-(4-(\mathrm{N}-$ pyrimidin-2ylsulfamoyl)phenyl)butanamide (19b) 
Yield (82\%), reddish brown powder, m.p. 213-215 ${ }^{\circ} \mathrm{C}$; IR (KBr): $v / \mathrm{cm}^{-1}$ b. 3320 (3NH), 1697 (C=O, amidic), $1662(\mathrm{C}=\mathrm{O}), 1562(\mathrm{~N}=\mathrm{N}) ;{ }^{1} \mathrm{H}$ NMR (DMSO- $\left.d_{6}\right) \delta(\mathrm{ppm})$ : $1.77\left(\mathrm{~s}, 3 \mathrm{H}, \mathrm{CH}_{3}\right), 2.50\left(\mathrm{~s}, 3 \mathrm{H}, \mathrm{CH}_{3}\right), 2.68\left(\mathrm{~s}, 3 \mathrm{H}, \mathrm{CH}_{3}\right)$, 7.47 (d, 2H Ar-H), 7.63 (d, 2H Ar-H), 6.87 and 7.20 (m, $3 \mathrm{H}, \mathrm{Ar}-\mathrm{H}$ ), 8.80 (s, H, NH), 9.00 (s, H, NH), 10.19 (s, H, $\mathrm{NH}) ; \mathrm{MS} m / z(\%) 548\left(\mathrm{M}^{+}, 32.27\right), 469$ (17.86), 391 (24.54), 314 (69.33), 202 (61.47), 98 (71.27), 79 (56.84), 76 (81.49); Anal. calcd. for $\mathrm{C}_{25} \mathrm{H}_{24} \mathrm{~N}_{8} \mathrm{O}_{5} \mathrm{~S}$ (548.16): C, 54.74; H, 4.41; N, 20.43. Found: C, 54.71; H, 4.33; N, 20.41 .

(E)-3-Oxo-N-(4-(N-pyrimidin-2-ylsulfamoyl)phenyl)-2-(2-(4-sulfamoylphenyl)hydrazono)butanamide (19c)

Yield (82\%), yellow powder, m.p. $162-165{ }^{\circ} \mathrm{C}$; IR (KBr): $v / \mathrm{cm}^{-1} 3453\left(\mathrm{NH}_{2}\right)$, b. $3250(3 \mathrm{NH}), 1703(\mathrm{C}=\mathrm{O}$, amidic), $1667(\mathrm{C}=\mathrm{O}), 1551(\mathrm{~N}=\mathrm{N}) ;{ }^{1} \mathrm{H} \mathrm{NMR}\left(\mathrm{DMSO}-d_{6}\right) \delta$ (ppm): 2.23 (s, 3H, $\left.\mathrm{CH}_{3}\right), 3.95\left(\mathrm{~s}, 2 \mathrm{H}, \mathrm{NH}_{2}\right), 7.62$ and 7.59 (dd, 4H, Ar-H), 7.82 (d, 2H Ar-H), 7.93 (d, 2H, Ar-H), 7.65-8.39 (m, 3H, Ar-H of pyrimidine), 8.82 (s, H, NH), 9.04 (s, H, NH), 10.16 (s, H, NH); MS m/z (\%) $517\left(\mathrm{M}^{+}\right.$, 47.37), 438 (26.41), 359 (62.64), 171 (74.91), 158 (69.23), 99 (84.16), 79 (69.65), 77 (85.22); Anal. calcd. for $\mathrm{C}_{20} \mathrm{H}_{19} \mathrm{~N}_{7} \mathrm{O}_{6} \mathrm{~S}_{2}$ (517.08): C, 46.41; H, 3.70; N, 18.94. Found: C, 46.53; H, 3.73; N, 18.89 .

(E)-3-Oxo-N-(4-(N-pyrimidin-2-ylsulfamoyl)phenyl)-2(2-(4-(N-thiazol-2-ylsulfamoyl)phenyl)hydrazono)butanamide (19d)

Yield (84\%), dark brown powder, m.p. charring at $300{ }^{\circ} \mathrm{C}$; IR (KBr): $v / \mathrm{cm}^{-1}$ b. $3261(4 \mathrm{NH}), 1723(\mathrm{C}=\mathrm{O}$, amidic), $1663(\mathrm{C}=\mathrm{O}), 1580(\mathrm{~N}=\mathrm{N}) ;{ }^{1} \mathrm{H}$ NMR (DMSO- $\left.d_{6}\right) \delta$ (ppm): 2.23 (s, 3H, $\mathrm{CH}_{3}$ ), 6.51 (s, $\mathrm{H}, \mathrm{CH}$ of thiazole ring), 7.14 (s, H, CH of thiazole ring), 6.81 and 7.34 (dd, $4 \mathrm{H}, \mathrm{Ar}-$ $\mathrm{H}), 7.82$ (d,2H Ar-H), 7.93 (d,2H Ar-H), 7.65-8.39 (m, $3 \mathrm{H}, \mathrm{Ar}-\mathrm{H}$ of pyrimidine), 8.82 (s, H, NH), 9.04 (s, H, NH), 10.16 (s, H, NH), 10.35 (s, H, NH); MS m/z (\%) $600\left(\mathrm{M}^{+}\right.$, 74.42), 442 (25.33), 254 (12.03), 171 (16.74), 158 (62.48), 156 (43.64), 85 (82.09), 78 (68.96); Anal. calcd. for $\mathrm{C}_{23} \mathrm{H}_{20} \mathrm{~N}_{8} \mathrm{O}_{6} \mathrm{~S}_{3}$ (600.07): C, 45.99; H, 3.36; N, 18.66. Found: C, 45.93; H, 3.33; N, 18.69 .

(E)-2-(2-(Benzo[d]thiazol-2-yl)hydrazono)-3-oxo-N-(4(N-pyrimidin-2-ylsulfamoyl)phenyl)butanamide (19e)

Yield (74\%), brown powder, m.p. charring at 200 ${ }^{\circ} \mathrm{C}$; IR (KBr): $v / \mathrm{cm}^{-1}$ b. 3341 (3NH), 1714 (C=O, amidic), $1669(\mathrm{C}=\mathrm{O}), 1571(\mathrm{~N}=\mathrm{N}) ;{ }^{1} \mathrm{H}$ NMR (DMSO- $\left.d_{6}\right) \delta(\mathrm{ppm})$ : 2.23 (s, 3H, $\mathrm{CH}_{3}$ ), 7.82 (d, 2H, Ar-H), 7.93 (d, 2H, Ar-H), 7.65-8.39 (m, 7H, Ar-H of pyrimidine and benzene rings), 8.82 (s, H, NH), 9.04 (s, H, NH), 10.16 (s, H, NH); MS $m / z(\%) 495\left(\mathrm{M}^{+}, 42.74\right), 337$ (36.75), 261 (26.82), 249 (29.35), 149 (15.77), 135 (46.44), 99 (75.24), 79 (62.96), 77 (88.57); Anal. calcd. for $\mathrm{C}_{21} \mathrm{H}_{17} \mathrm{~N}_{7} \mathrm{O}_{4} \mathrm{~S}_{2}$ (495.08): C, 50.90; H, 3.46; N, 19.79. Found: C, 50.93; H, $3.33 ; \mathrm{N}, 19.69$.
(E)-2-(2-(1H-Imidazol-2-yl)hydrazono)-3-oxo-N-(4-(Npyrimidin-2-ylsulfamoyl)phenyl)butanamide(19f)

Yield (85\%), brown powder, m.p. charring at $232^{\circ} \mathrm{C}$; IR (KBr): $v / \mathrm{cm}^{-1}$ b. 3441 (4NH), 1706 (C=O, amidic), 1664 $(\mathrm{C}=\mathrm{O}), 1563(\mathrm{~N}=\mathrm{N}) ;{ }^{1} \mathrm{H}$ NMR (DMSO- $\left.d_{6}\right) \delta(\mathrm{ppm}): 2.23$ (s, 3H, $\left.\mathrm{CH}_{3}\right), 7.03$ and $7.05(\mathrm{~d}, 2 \mathrm{H}, 2 \mathrm{CH}), 7.82(\mathrm{~d}, 2 \mathrm{H}, \mathrm{Ar}-$ $\mathrm{H}), 7.93$ (d, 2H, Ar-H), 7.65-8.39 (m, 3H, Ar-H of pyrimidine), 8.82 (s, H, NH), 9.04 (s, H, NH), 10.16 (s, H, NH), 12.81 (s, H, NH of imidazole ring); $\mathrm{MS} m / z$ (\%) $433\left(\mathrm{M}^{+}+5\right.$, 36.00), 231 (41.10), 202 (99.90), 186 (39.10); Anal. calcd. for $\mathrm{C}_{17} \mathrm{H}_{16} \mathrm{~N}_{8} \mathrm{O}_{4} \mathrm{~S}$ (428.10): C, 47.66; $\mathrm{H}, 3.76 ; \mathrm{N}, 26.15$. Found: C, 47.63; H, 3.83; N, 26.39.

(E)-2-(2-(1H-Benzo[d]imidazol-2-yl)hydrazono)-3-oxo$\mathrm{N}$-(4-(N-pyrimidin-2-ylsulfamoyl)phenyl)butanamide (19g)

Yield (78\%), pale brown powder, m.p. charring at 251 ${ }^{\circ} \mathrm{C}$; IR (KBr): v/ $\mathrm{cm}^{-1}$ b. 3267 (4NH), 1700 (C=O, amidic), $1668(\mathrm{C}=\mathrm{O}), 1573(\mathrm{~N}=\mathrm{N}) ;{ }^{1} \mathrm{H}$ NMR (DMSO- $\left.d_{6}\right) \delta(\mathrm{ppm})$ : $2.23\left(\mathrm{~s}, 3 \mathrm{H}, \mathrm{CH}_{3}\right), 7.82$ and 7.93 (dd, $\left.4 \mathrm{H}, \mathrm{Ar}-\mathrm{H}\right), 7.65-8.39$ ( $\mathrm{m}, 7 \mathrm{H}, \mathrm{Ar}-\mathrm{H}$ of pyrimidine and benzene rings), 8.82 (s, H, $\mathrm{NH}), 9.04$ (s, H, NH), 10.16 (s, H, NH), 10.81 (s, H, NH); MS $m / z(\%) 483\left(\mathrm{M}^{+}+5,1.20\right), 283$ (87.60), 267 (99.90), 171 (82.40); Anal. calcd. for $\mathrm{C}_{21} \mathrm{H}_{18} \mathrm{~N}_{8} \mathrm{O}_{4} \mathrm{~S}$ (478.1): C, 52.71; $\mathrm{H}$, 3.79; N, 23.42. Found: C, 52.64; H, 3.83; N, 23.35.

(E)-3-Oxo-N-(4-(N-pyrimidin-2-ylsulfamoyl)phenyl)-2(2-(4-(N-pyrimidin-2-ylsulfamoyl)phenyl)hydrazono) butanamide (19h)

Yield (83\%), black powder, m.p. charring at $251{ }^{\circ} \mathrm{C}$; IR (KBr): $v / \mathrm{cm}^{-1}$ b. $3339(4 \mathrm{NH}), 1731(\mathrm{C}=\mathrm{O}$, amidic), 1665 $(\mathrm{C}=\mathrm{O}), 1571(\mathrm{~N}=\mathrm{N}) ;{ }^{1} \mathrm{H}$ NMR (DMSO- $\left.d_{6}\right) \delta(\mathrm{ppm}): 2.23$ (s, 3H, $\left.\mathrm{CH}_{3}\right), 7.79-7.96$ (dd, 8H, Ar-H), 7.65-8.39 (m, 6H, Ar-H of pyrimidine), 8.82 (s, 2H, 2NH), 9.04 (s, H, NH), 10.16 (s, H, NH); MS m/z (\%) $595\left(\mathrm{M}^{+}, 28.63\right), 249$ (59.26), 157 (74.59), 101 (48.42), 98 (64.88), 98 (49.04), 93 (59.69); Anal. calcd. for $\mathrm{C}_{24} \mathrm{H}_{21} \mathrm{~N}_{9} \mathrm{O}_{6} \mathrm{~S}_{2}$ (595.11): C, 48.40; H, 3.55; N, 21.16. Found: C, 48.51; H, 3.43; N, 21.33.

\section{3. Cytotoxicity Activity}

RPMI-1640 medium (Sigma Co., St. Louis, USA), Foetal Bovine serum (GIBCO, UK), and the cell lines from ATCC were used.

The cytotoxic activities of the prepared sulfonamides were examined versus HepG2, WI-38, MCF-7, and VERO, carried out according to the previously reported procedure. $^{16}$

\section{Results and Discussion}

\section{1. Chemistry}

From the above reports in sulfonamide chemistry, we prepared some new heterocyclic compounds containing sulfonamide moiety to evaluate their biological activi- 
ties. Condensation of compound $\mathbf{1}$ with acid anhydrides, namely phthalic anhydride, 3-nitrophthalic anhydride, and 4-nitrophthalic anhydride in refluxing $\mathrm{N}, \mathrm{N}$-dimethylformamide containing a few drops of triethylamine yielded sulfonamides $\mathbf{2}-\mathbf{4}$. The infrared spectra of sulfonamides 2-4, in general, displayed no absorption of $\mathrm{NH}_{2}$ at 3426 $\mathrm{cm}^{-1}$ of sulfonamide $\mathbf{1}$, and instead, appeared new bands within the region $1631-1721 \mathrm{~cm}^{-1}$ due to carboximide groups. The MS spectra of sulfonamides $\mathbf{2 - 4}$ offered the molecular weight at $m / z 378\left(\mathrm{M}^{+}-2\right)$ and at $m / z 423\left(\mathrm{M}^{+}-\right.$ 2) corresponding to molecular formulae $\mathrm{C}_{18} \mathrm{H}_{12} \mathrm{~N}_{4} \mathrm{O}_{4} \mathrm{~S}$ and/or $\mathrm{C}_{18} \mathrm{H}_{11} \mathrm{~N}_{5} \mathrm{O}_{6} \mathrm{~S}$, respectively.

Similarly, condensation of compound 1 with 3,4,5,6-tetrabromophthalic anhydride, and 1,2,4-benzene- tricarboxylic anhydride (trimellitic anhydride) in boiling $\mathrm{N}, \mathrm{N}$-dimethylformamide including a few small drops of triethylamine gave sulfonamides $\mathbf{5}$ and $\mathbf{6}$, respectively. Structures $\mathbf{5}$ and $\mathbf{6}$ were elucidated by different analyses. The infrared spectra of compounds 5 and $\mathbf{6}$ exhibited bands at 1720,1632 , and $1330 \mathrm{~cm}^{-1}$ due to CO groups and $\mathrm{SO}_{2}$ functional groups. The MS spectra of $\mathbf{5}$ and $\mathbf{6}$ offered molecular weight at $m / z 691\left(\mathrm{M}^{+}\right)$and $426\left(\mathrm{M}^{+}+2\right)$, respectively

On the other hand, heating of sulfonamide 1 with pyromellitic anhydride in ethanol with a few drops of triethylamine in a molar ratio 1:2 gave pyromellitimide 7 . The infrared spectrum of compound 7 showed a similar picture to that of $\mathbf{5}$ and $\mathbf{6}$. The MS spectrum of $\mathbf{7}$ gave molecular<smiles>O=C1c2ccccc2C(=O)N1[Ga]</smiles><smiles>CCOC(=O)c1ccccc1C(=O)O</smiles><smiles>CCN1C(=O)c2cccc([N+](=O)[O-])c2C1=O</smiles>

3<smiles>O=C1c2ccc([N+](=O)[O-])cc2C(=O)N1[Al]</smiles><smiles></smiles><smiles>O=C1OC(=O)c2c(Br)c(Br)c(Br)c(Br)c21</smiles>

5<smiles>Nc1ccc(OS(=O)(=O)Nc2ncccn2)cc1</smiles><smiles>O=C(O)c1ccc2c(c1)C(=O)N([Al])C2=O</smiles><smiles>O=c1[nH]c2ccccc2c(=O)o1</smiles><smiles>O=c1[nH]c2ccccc2c(=O)n1[Ga]Cl</smiles>

8<smiles>Cc1ccc(OS(=O)(=O)Nc2ncccn2)cc1</smiles>

Scheme 1. Synthesis of sulfonamide derivatives 2-8 
<smiles>CCOC(=O)C(=COCCCCCCO)C(=O)OCC</smiles>

1<smiles>CCOC(=O)C(=CNc1ccc(OS(=O)(=O)Nc2ncccn2)cc1)OCC</smiles>

9<smiles></smiles>

Scheme 2. Synthesis of acid hydrazide $\mathbf{1 1}$

weight at $m / z 680\left(\mathrm{M}^{+}-2\right)$. In addition, stirring of sulfonamide 1 with isatoic anhydride in ethanol including a few small drops of triethylamine at room temperature yielded compound 8 . The infrared spectrum of sulfonamide derivative 8 displayed band at $v 1679 \mathrm{~cm}^{-1}$ corresponding to carboximide functional group. The MS spectrum gave additional confirmation for the correct structure of sulfonamide 8 as it gave a molecular ion peak at $m / z 393\left(\mathrm{M}^{+}-2\right)$.

The starting material $\mathbf{1}$ was refluxed with diethyl 2-(ethoxymethylene)malonate in a mixture of $\mathrm{N}, \mathrm{N}$-dimethylformamide and ethanol (1:1 ratio) to give compound 9 in high yield. The infrared spectrum of 9 displayed a band at $1689 \mathrm{~cm}^{-1}$ for ester carbonyl functional group, and an absorption frequency at $3373 \mathrm{~cm}^{-1}$ corresponding to $\mathrm{NH}$ group. The MS spectum of 9 offered molecular weight at $m / z 422\left(\mathrm{M}^{+}+2\right)$ confirming the formula
$\mathrm{C}_{18} \mathrm{H}_{20} \mathrm{~N}_{4} \mathrm{O}_{6} \mathrm{~S}$. Cyclization reaction of compound 9 was performed by boiling in diphenyl ether to give ethyl 4-oxo-6-( $N$-pyrimidin-2-ylsulfamoyl)-1,4-dihydroquinoline-3-carboxylate (10) in acceptable yield. The infrared spectrum of 10 displayed the distinctive bands at $\nu 3219$, 1681,1623 , and $1516 \mathrm{~cm}^{-1}$ due to $\mathrm{NH}, \mathrm{C}=\mathrm{O}$ of ester, $\alpha, \beta$-unsaturated $\mathrm{C}=\mathrm{O}$ and $\mathrm{C}=\mathrm{C}$ groups. Its ${ }^{1} \mathrm{H}$ NMR spectrum revealed a triplet signal at $\delta 1.30 \mathrm{ppm}\left(\mathrm{CH}_{3}\right)$, a quartet signal at $\delta 4.31 \mathrm{ppm}\left(\mathrm{CH}_{2}\right)$, a $\mathrm{D}_{2} \mathrm{O}$ exchangeable two $\mathrm{NH}$ at $\delta 8.94$ and $9.17 \mathrm{ppm}$ as singlet signals, and a singlet signal for $\mathrm{C}_{2}-\mathrm{H}$ of quinoline ring at $\delta 7.24 \mathrm{ppm}$ besides the aromatic hydrogens of quinoline and pyrimidine rings at $\delta$ 7.69-7.85 ppm. In addition, the MS offered another confirmation for compound $\mathbf{1 0}$ as it gave its molecular weight at $\mathrm{m} / \mathrm{z} 375\left(\mathrm{M}^{+}+1\right)$ corresponding to the formula $\mathrm{C}_{16} \mathrm{H}_{14} \mathrm{~N}_{4} \mathrm{O}_{5} \mathrm{~S}$.<smiles>O=C(NNC(=S)S)c1c[nH]c2ccc(OS(=O)(=O)Nc3ncccn3)cc2c1=O</smiles><smiles>O=c1c(-c2n[nH]c(=S)o2)c[nH]c2ccc(OS(=O)(=O)Nc3ncccn3)cc12</smiles>

Scheme 3. Synthesis of sulfonamide derivative $\mathbf{1 3}$ 
<smiles>Cc1nnc(-c2c[nH]c3ccc(OS(=O)(=O)Nc4ncccn4)cc3c2=O)o1</smiles>

13<smiles>O=c1c(-c2n[nH]c(=S)o2)c[nH]c2ccc(OS(=O)(=O)Nc3ncccn3)cc12</smiles><smiles>C/C=C\CCSc1nnc(-c2c[nH]c3ccc(OS(=O)(=O)Nc4ncccn4)cc3c2=O)o1</smiles>

14<smiles>[Y]COOC(C)=O</smiles>

15

Scheme 4. Synthesis of sulfonamide derivative $\mathbf{1 4}$ and $\mathbf{1 5}$

Boiling 10 with hydrazine hydrate in ethanol/DMF solution (1:1 ratio) afforded the corresponding acid hydrazide 11. The infrared spectrum of $\mathbf{1 0}$ displayed bands at $v 3435$ and $3360 \mathrm{~cm}^{-1}$ due to $\mathrm{NHNH}_{2}$ group, besides the presence of amide carbonyl group at $v 1685 \mathrm{~cm}^{-1}$

Reaction of hydrazide $\mathbf{1 1}$ with carbon disulphide in refluxing pyridine afforded the thioic acid $\mathbf{1 2}$. The infrared spectrum displayed absorption bands at $v 3287,3214,1688,1625$, and $1332 \mathrm{~cm}^{-1}$ due to stretching vibration of $\mathrm{NH}$, amide $\mathrm{CO}$, $\alpha, \beta$-unsaturated ketone, and $\mathrm{C}=\mathrm{S}$ groups. The $\mathrm{MS}$ spectrum of compound 12 offered a molecular weight at $m / z 432\left(\mathrm{M}^{+}-4\right)$.

When thioic acid derivative $\mathbf{1 2}$ was heated under reflux in $\mathrm{N}, \mathrm{N}$-dimethylformamide containing a few drops of triethylamine, sulfonamide derivative $\mathbf{1 3}$ was afforded. The infrared spectrum of $\mathbf{1 3}$ showed a strong band at $\boldsymbol{v}$ $1332 \mathrm{~cm}^{-1}$ due to $\mathrm{C}=\mathrm{S}$ functional group, and a weak band at $v 2615 \mathrm{~cm}^{-1}$ due to $\mathrm{SH}$ vibration in a tautomeric mixture as well. The MS spectrum displayed its molecular weight at $m / z 402\left(\mathrm{M}^{+}\right)$.

It has been found that compound $\mathbf{1 3}$, when subjected to react with methyl iodide in sodium hydroxide solution, afforded the $\mathrm{SCH}_{3}$ derivative 14 (Scheme 4), while when 13 reacted with formaldehyde and para-toluidine it afforded the Mannich base 15 (Scheme 4); such reactions were carried out to indicate the thiol-thione tautomerism.

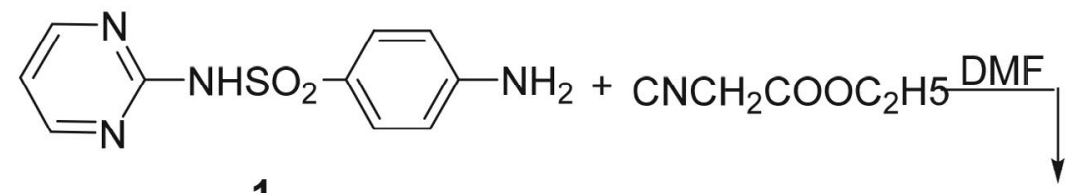

\section{1}

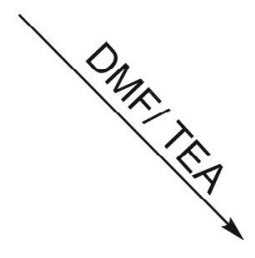<smiles>N#CCC(=O)Nc1ccc(OS(=O)(=O)Nc2ncccn2)cc1</smiles>

16

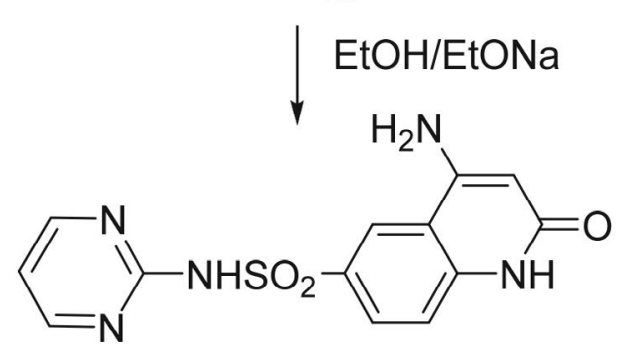


We have observed that thiol-thione tautomerism exists in compounds 14 and $15 .{ }^{1} \mathrm{H}$ NMR of compound 14 showed signal at $1.96 \mathrm{ppm}$ referred to $\mathrm{SCH}_{3}$ protons, while the infrared spectrum of 15 offered a band at $v 1267 \mathrm{~cm}^{-1}$ due to $\mathrm{C}=\mathrm{S}$.

Moreover, it was found that ethyl acetoacetate reacted with compound 1 in refluxing $N, N$-dimethylformamide including a few small drops of trimethylamine to give the acetamide derivative $\mathbf{1 6}$.

The infrared spectrum of sulfonamide $\mathbf{1 6}$ gave 3 lines at 1647,2155 , and $3357 \mathrm{~cm}^{-1}$ characteristic to amide CO, $\mathrm{CN}$, and $\mathrm{N}-\mathrm{H}$ groups. Its ${ }^{1} \mathrm{H}$ NMR spectrum showed signals at $\delta 4.25 \mathrm{ppm}$ corresponding to $\mathrm{CH}_{2}$ protons and 11.19 ppm corresponding to amide $\mathrm{NH}$ group. The MS spectrum showed additional confirmation to validate the compound as it showed the molecular weight at $\mathrm{m} / z 317$ $\left(\mathrm{M}^{+}\right)$. When compound $\mathbf{1 6}$ was heated in ethanol containing sodium ethoxide, a cyclization occured forming the corresponding 4-amino-2-oxo- $N$-(pyrimidin-2-yl)-1,2-dihydroquinoline-6-sulfonamide (17). Structure 17 was established based on its correct spectral and elemental analyses. The infrared spectrum displayed lines at 3357, 1713, 1651 , and $1584 \mathrm{~cm}^{-1}$ corresponding to $\mathrm{NH}$, amidic carbonyl, $\alpha, \beta$-unsaturated ketone, and $\mathrm{C}=\mathrm{C}$ groups. ${ }^{1} \mathrm{H} \mathrm{NMR}$ also revealed a very characteristic signal at $\delta 4.82 \mathrm{ppm}$ due to $\mathrm{NH}_{2}$ functional group. The MS spectrum offered a molecular weight at $m / z 312\left(\mathrm{M}^{+}-5\right)$. Structure 17 was confirmed also chemically by an alternative preparation. Thus, heating of sulfonamide $\mathbf{1}$ in the presence of $N, N$-dimethylformamid and triethylamine afforded compound conforming in all its properties (m.p., TLC, and infrared spectrum) to the sulfonamide derivative $\mathbf{1 7}$.

When sulfonamide 1 reacted with ethyl acetoacetate in dry boiling xylene, it gave the acyclic intermediate 3-oxo- $N$-(4-( $N$-pyrimidin-2-ylsulfamoyl)phenyl)butanamide (18). The infrared spectrum of compound 18 offered new absorption bands at 1689 and $1725 \mathrm{~cm}^{-1}$ corresponding to carbonyl of $\mathrm{COOR}$ and amide $\mathrm{C}=\mathrm{O}$ functional groups, while the ${ }^{1} \mathrm{H}$ NMR spectrum of sulfonamide $\mathbf{1 8}$ displayed a characteristic signal at $\delta 2.31 \mathrm{ppm}$ as a singlet for $\mathrm{CH}_{3}$ protons, $\delta 3.33$ ppm signal for $\mathrm{CH}_{2}$ protons besides the aromatic protons of pyrimidine ring and benzene ring at $\delta 7.45-8.21 \mathrm{ppm}$. The mass spectrometry measurement gave $m / z 335\left(\mathrm{M}^{+}-1\right)$ conforming to the molecular weight of compound $\mathbf{1 8}$.

Coupling of sulfonamide 18 with aromatic amine diazonium salts (namely 2-aminothiazole diazonium salt, 4-aminoantipyrine diazonium salt, sulfanilamide diazonium salt and sulfathiazole diazonium salt, 2-aminobenzthiazole diazonium salt, 2-aminoimidazole diazonium salt, 2-aminobenzimidazole diazonium salt, and sulfadiazine diazonium salt) in pyridine at $0-5{ }^{\circ} \mathrm{C}$ gave the corresponding hydrazono compounds $\mathbf{1 9 a}-\mathbf{h}$. The spectral and elemental analyses are in harmony with the suggested compound structures. Thus, ${ }^{1} \mathrm{H}$ NMR spectrum of compound 19a displayed the disappearance of protons at $\delta 3.33 \mathrm{ppm}$

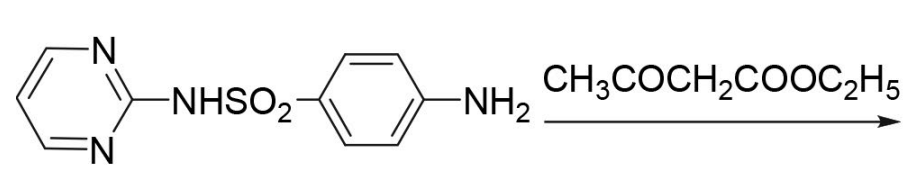

1

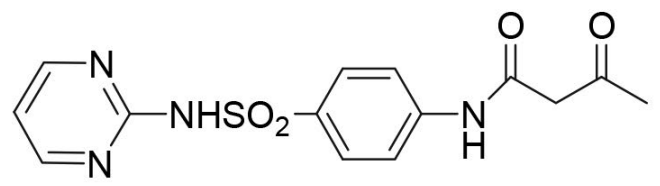

18

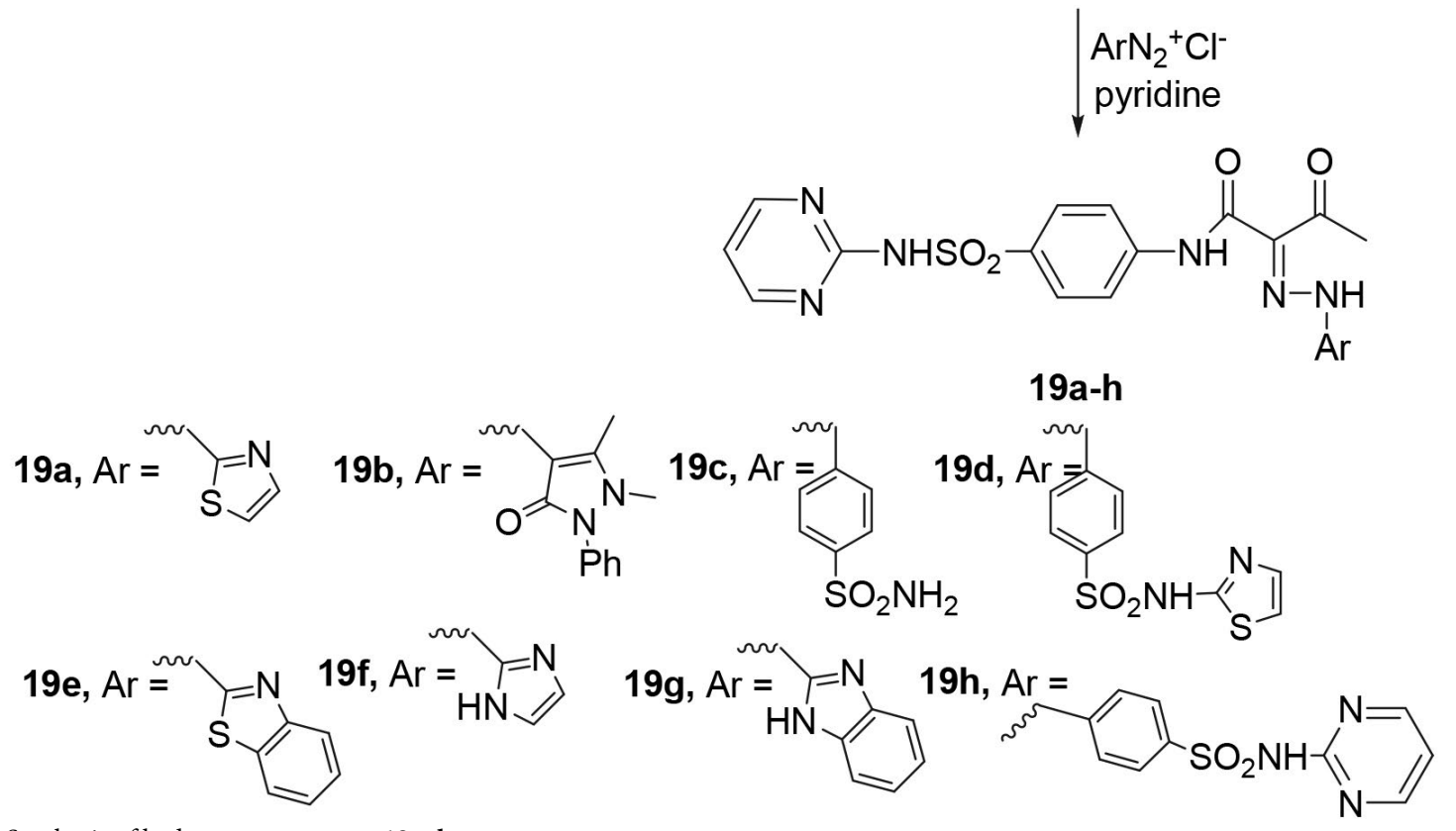

Scheme 6. Synthesis of hydrazono structures $19 \mathbf{a}-\mathbf{h}$ 
<smiles>CC(=O)/C(=N\N[Ga])C(=O)Nc1ccc(OS(=O)(=O)c2ccc(Nc3ccc(S(=O)(=O)Nc4ncccn4)cc3)nc2)cc1</smiles>

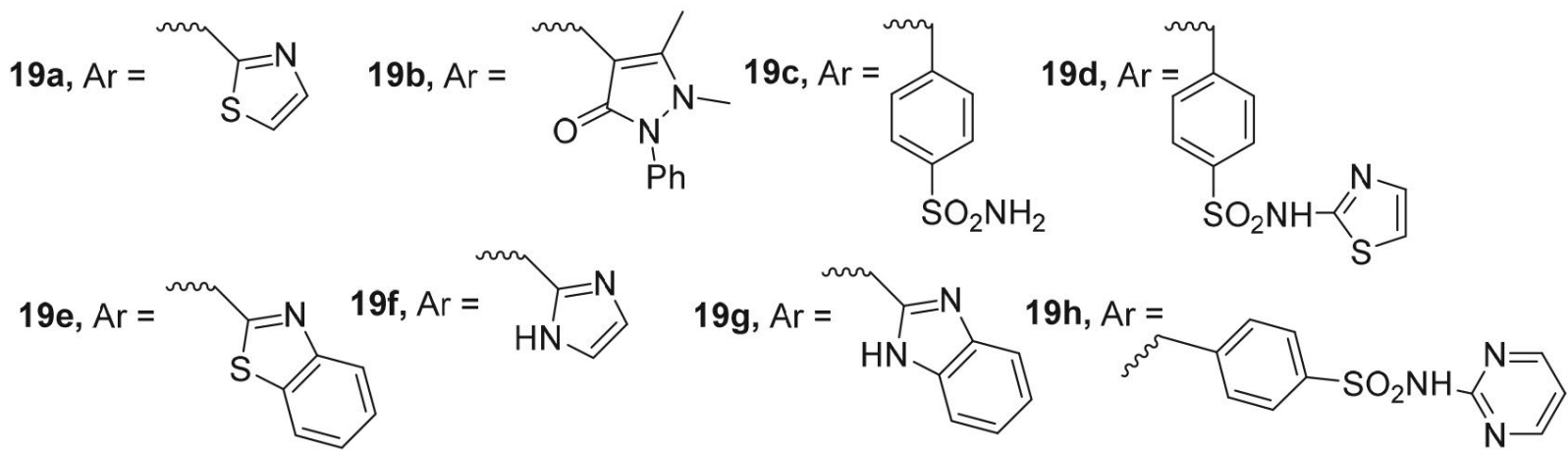

Scheme 7. Intramolecular hydrogen bonding is in support of the hydrazone moieties in sulfonamides 19a-h

due to $\mathrm{CH}_{2}$ and the appearance of two doublet signals at $\delta$ 6.57 and $7.04 \mathrm{ppm}$ due to two $\mathrm{CH}$ of thiazole ring. Similarly, the ${ }^{1} \mathrm{H}$ NMR of compound $19 b$ showed two singlet signals appeared at $\delta 1.77$ and $2.68 \mathrm{ppm}$ for $\mathrm{CH}_{3}$ protons. The infrared spectrum of sulfonamides $\mathbf{1 9} \mathbf{a}-\mathbf{h}$ in general exhibited a band in the area $3200-3450 \mathrm{~cm}^{-1}$ due to $\mathrm{NH}$ vibration of the hydrazone moiety and a band at 1550-1580 $\mathrm{cm}^{-1}$ due to the azo form in its tautomeric equilibrium as well. Such shift of NH band was notified by Ramirez and Kerby ${ }^{17}$ for these hydrazone derivatives, this being due to the intra-molecular hydrogen bonding as depicted in structure A (Scheme 7). These facts indicate that sulfonamide derivatives 19a-h offer confirmation for intramolecular hydrogen bonding which supports the hydrazone compounds. Infrared spectra of sulfonamides $19 \mathbf{a}-\mathbf{h}$ displayed bands at $1662-1731 \mathrm{~cm}^{-1}$ corresponding to vibration of $\mathrm{C}=\mathrm{O}$ functions.

Among the structural factors that make minimizing of the vibration of carbonyl functional group are conjugation and hydrogen bonding. Albeit, even when allowance is exhibited for conjugation, the carbonyl frequencies of the sulfonamides calculated are still much smaller than those in $\alpha, \beta$-unsaturated ketones. This marked variance suggests that the carbonyl function of these sulfonamides should participate in hydrogen bonding in the solid state, as demonstrated by the suggested compound $\mathbf{A}$. The ultraviolet spectra of the diazonium coupling products gave an extra confirmation that sulfonamides are in the tautomeric equilibrium with monohydrazones. Maximum of the dyes displayed 4 absorption bands at 196-438 nm. The comparatively low variance in $\lambda_{\max }$ may correspond to the polarity variation of the band due to solvent actions corresponding to the common solvent effect. ${ }^{18}$ It has been seen that the ultraviolet spectra of monophenylazo structures differ from those of monophenylhydrazones. The azo structures commonly display two bands at $400-410$ and $290-300 \mathrm{~nm}$ due to $n-\pi^{*}$ and $\pi-\pi^{*}$ transitions, respectively. ${ }^{19}$

Moreover, monophenylhydrazones display tree bands at $220-230,250-280$ and $330-390 \mathrm{~nm}$ regions. The ultraviolet spectra of sulfonamides $\mathbf{1 9} \mathbf{a}-\mathbf{h}$ can be explained in terms of the tautomeric mixture. These dyes show four bands, of these, the medium and high wavelength bands appear to be dependent on the type of the polar substituents in the arylazo function, while the low wavelength bands is not affected. The UV spectral data show that both electron withdrawing groups and electron donating groups shift the absorption maxima to longer wavelengths. Moreover, it showed that the presence of this has not caused any observed higher or lower values of $\lambda_{\max }$ in the apparent region and $\log \varepsilon$ has stayed nearly constant. This does point to the presence also of hydrazine structure where the resonance actions with the functions in the diazo structure are lower due to steric effects. In addition, the structure of sulfonamides 19a-h was elucidated by mass spectra which displayed the correct molecular weight.

\section{2. Pharmacology}

\section{2. 1. Cytotoxicity}

The use of heterocyclic structures has a significant role in the treatment of cancer and in its removal plans. ${ }^{20}$ Heterocycles are generally used as scaffolds on which 
pharmacophores are coordinated to give effective and electic drugs. This is especially correct for five membered-ring heterocyclic compounds, ${ }^{21}$ which serve as the core components of a huge number of compounds that have a broad motivating range of biological action. ${ }^{22-24}$ The goal of this study was to prepare new drugs for anticancer elucidation as a trial to give novel antitumor agents of a maximum action and minimize side effects. In this paper, the chosen sulfonamides regarding to pyridine, pyrimidine, oxadiazole, and azo compounds were elucidated and screened in vitro for prevention of the growth of HepG2 (human hepatocellular liver carcinoma cell lines), WI 38 (human lung fibroblasts), VERO (cell line was initiated from the kidney of a normal adult African green monkey), and MCF-7 (breast cancer cell lines) were compared with the known anticancer drug 5-fluorouracil (5$\mathrm{Fu}$ ) and as a trial to get more potent agents with lower toxicity. The collected data are presented as the concentration of sulfonamides that made $50 \%$ inhibition of cells growth $\left(\mathrm{IC}_{50}\right)$.

The in vitro elucidation showed that some of the examined sulfonamides displayed (and also that all the modern prepared structures revealed) a certain action against tumor cell lines examined, although the action was commonly maximal towards HepG2 cancer line than the breast cancer one. Compounds $\mathbf{3}$ and $\mathbf{5}$ showed an effective antitumor action versus the four tumor cell lines examined $\left(\mathrm{IC}_{50}=11\right.$ and 12.4 against $\mathrm{HepG} 2$ and $\mathrm{IC}_{50}=7.3$ and 9.32 against VERO, respectively) in contrast to the effective anticancer drug 5-fluorouracil used as the reference standard. So, we found that all examined sulfonamides including electron withdrawing groups $\left(\mathrm{Br}\right.$ or $\left.\mathrm{NO}_{2}\right)$ in the

Table 1: Cytotoxicity $\left(\mathrm{IC}_{50}\right)$ of examined sulfonamides on various cell lines ${ }^{\mathrm{a}}$

\begin{tabular}{lcccc}
\hline Compound No. & \multicolumn{4}{c}{ IC $_{\mathbf{5 0}}(\boldsymbol{\mu g} / \mathbf{m L})^{\mathbf{a}}$} \\
& HepG2 & WI-38 & VERO & MCF-7 \\
\hline $5-\mathrm{Fu}$ & 8.6 & 3.2 & 6.5 & 2.3 \\
$\mathbf{2}$ & 88 & 74 & 90 & 85.5 \\
$\mathbf{3}$ & $\mathbf{1 1}$ & $\mathbf{1 7 . 1}$ & 7.3 & $\mathbf{1 0 . 5}$ \\
$\mathbf{4}$ & 23.2 & 33.7 & 30.3 & 26.1 \\
$\mathbf{5}$ & $\mathbf{1 2 . 4}$ & $\mathbf{2 0}$ & $\mathbf{9 . 3 2}$ & $\mathbf{1 2 . 5}$ \\
$\mathbf{6}$ & 26 & 40.3 & 38.1 & 45 \\
$\mathbf{7}$ & 102 & 120 & 100 & 200 \\
$\mathbf{8}$ & 29.5 & 27.6 & 36.5 & 29.9 \\
$\mathbf{1 9 a}$ & 42 & 46.1 & 43.1 & 42.2 \\
$\mathbf{1 9 b}$ & 65.2 & 70.3 & 86 & 71.6 \\
$\mathbf{1 9 c}$ & 26 & 40.3 & 38.1 & 45.5 \\
$\mathbf{1 9 d}$ & $\mathbf{1 5 . 5}$ & $\mathbf{1 6 . 6}$ & $\mathbf{2 6 . 5}$ & $\mathbf{2 7 . 9}$ \\
$\mathbf{1 9 e}$ & 33.1 & 39.8 & 42.6 & 45.6 \\
$\mathbf{1 9 f}$ & 88.2 & 74 & 90 & 85.5 \\
$\mathbf{1 9 g}$ & 92 & 99.3 & 75.2 & 94.3 \\
$\mathbf{1 9 h}$ & $\mathbf{1 0 . 4}$ & $\mathbf{1 5 . 1}$ & $\mathbf{9 . 3}$ & $\mathbf{1 1 . 5}$ \\
\hline
\end{tabular}

${ }^{\mathrm{a}} \mathrm{IC}_{50}(\mu \mathrm{g} / \mathrm{mL})$ : 1-10 (very strong), 11-25 (strong), 26-50 (moderate), 51-100 (weak), 100-200 (very weak), above 200 (non cyotoxic). 5 -FLU $=5$-Fluorouracil benzene ring of phthaliamide moiety displayed a higher to moderate action, so the real effects depend on the existence of thiazole or pyrimidine ring attached to sulfonamide group. It is obvious that compound 19d showed strong action against two various cell lines and compound 19h showed strong action versus four various cell lines corresponding to a long $\pi$-conjugated system joined to thiazole and pyrimidine rings, besides azo functional group and benzene ring. These outcomes demonstrate that variable molecular structure and orientation could enhance commonly observed antitumor action against the four examined cancer cells.

\section{Conclusion}

In the current research, fifteen sulfonamides were examined and most of them showed an antitumor action on four cancer cell lines. The most effective compounds 3 , 5, 19d and 19h showed eclectic effect on HepG2 cancer cell line. The gained data exhibit the necessity or further developments to facilitate the future research based on the antitumor potential of the examined sulfonamides.

\section{References}

1. A. A. Fadda, A. M. Khalil, M. M. El-Habbal, Pharmazie 1991, 46, 743-744.

2. A. E. Boyd, Diabetes 1988, 37, 847-850. DOI:10.2337/diabetes.37.7.847

3. C. T. Supuran, A. Scozzafava, Exp. Opin. Ther. Patents 2000, 10, 575-600. DOI:10.1517/13543776.10.5.575

4. C. T. Supuran, A. Scozzafava, Curr. Med. Chem. Immunol. Endocr. Metabol. Agents 2001, 1, 61-97.

DOI:10.2174/1568013013359131

5. C. W. Thornber, Chem. Soc. Rev. 1979, 8, 563-580. DOI:10.1039/cs9790800563

6. S. A. Rostom, Bioorg. Med. Chem. 2006, 14, 64756485.

DOI:10.1016/j.bmc.2006.06.020

7. M. S. Al-Said, M. M. Ghorab, M. S. Al-Dosari, M. M. Hamed, Eur. J. Med. Chem. 2011, 46, 201-207. DOI:10.1016/j.ejmech.2010.11.002

8. A. A. Fadda, E. M. Afsah, R S. Awad, Eur. J. Med. Chem. 2013, 60, 421-430. DOI:10.1016/j.ejmech.2012.11.017

9. A. A. Fadda, K. Elattar, Med. J. Chem. 2013, 2013, 10. DOI:10.1155/2013/928106

10. A. A. Fadda, H. A. Etman, A. A. Sarhan, Sherihan A. El-Hadidy, Phosphorus, Sulfur, Silicon Relat. Elem. 2010, 185, 526536. DOI:10.1080/10426500902839863

11. A. A. Fadda, M. A. Berghot, F. A. Amer, D. S. Badawy, Nesma M. Bayoumy, Arch. Pharm. Chem., Life Sci (Archiv der Pharmazie) 2012, 345, 378-355.

DOI:10.1002/ardp.201100335

12. Hanaa Abuo-Melha, A. A. Fadda, Spectrochimica Acta Part A: 
Molecular and Biomolecular Spectroscopy 2012, 89, 123-128. DOI:10.1016/j.saa.2011.12.054

13. A. A. Fadda, A. A.-H. Abdel-Rahman, W. A. El-Sayed, T. A. Zidan, F. A. Badria, Chem. Heterocyc. Compd. 2011, 47, 856864. DOI:10.1007/s10593-011-0847-4

14. A. A. Fadda, E. Abdel-Latif, R. E. El-Mekawy, Pharmacol. Pharm. 2012, 3, 148-157.

DOI:10.4236/pp.2012.32022

15. A. El-Shafei, A. A. Fadda, A. M. Khalil, T. A. E. Ameen, F. A. Badria, Bioorg. Med. Chem. 2009, 17, 5096-5105.

DOI:10.1016/j.bmc.2009.05.053

16. A. A. Fadda, A. M. El Defrawy; Sherihan A. El-Hadidy, Am. J. Org. Chem. 2012, 2(4), 87-96.

DOI:10.5923/j.ajoc.20120204.03

17. F. Ramirez, A. F. Kirby, J. Am. Chem. Soc. 1954, 76, 1037. DOI:10.1021/ja01633a034
18. A. E. Gilman, E. S. Stern, An introduction to electronic absorption spectroscopy in organic chemistry, 2nd ed., Edward Arnold Publisher Ltd, London 1957, pp. 302.

19. A. E. Gilman, E. S. Stern, Electronic absorption spectroscopy, Edward Arnold Publisher Ltd, London 1960, pp. 271.

20. S. Abu-Melha, Acta Chim. Slov. 2017, 64, 910-930.

21. T. Foud, C. Nielsen, L. Brunn, E. B. Pederson, Sc. J. Az. Med. Fac. (Girls) 1998, 19, 1173-1187.

22. E. H. Tawfik, K. S. Mohamed, H. M. Dardeer, A. A. Fadda, Acta Chim. Slov. 2018, 65, 787-794.

DOI:10.17344/acsi.2018.4294

23. E. H. EL-Sayed,A. A. Fadda, Acta Chim. Slov. 2018, 65, 853864. DOI:10.17344/acsi.2018.4506

24. S. Botros, O. M. Khalil, M. M. Kamal, Y. S. El-Dash, Acta Chim. Slov. 2017, 64, 102-116. DOI:10.17344/acsi.2016.2901

25. R. M. Mohareb, N. Y. M. Abo, F. O. Al-Farouk, Acta Chim. Slov. 2017, 64, 117-128. DOI:10.17344/acsi.2016.2920

\section{Povzetek}

Na osnovi izhodne spojine 4-amino- $N$-(pirimidin-2-il)benzensulfonamida (1) smo pripravili serijo substituiranih sulfadiazinskih spojin in preučili njihovo citotoksično in antitumorsko delovanje. Spojino 1 smo reagirali $\mathrm{z}$ različnimi reagenti in tako pripravili sulfadiazine 2-18 ter hidrazone 19a-h in preučili njihovo in vitro citotoksičnost na štiri rakave celične linije. Ugotovili smo, da so spojine $3,5,19 \mathrm{~d}$ in $19 \mathrm{~h}$ aktivne proti preiskovanim rakavim celicam.

Except when otherwise noted, articles in this journal are published under the terms and conditions of the Creative Commons Attribution 4.0 International License 